\title{
Quantum Optics of Quantum Emitters in the Near-Field of a Plasmonic Nanoparticle
}

\author{
Victor Zadkov ${ }^{1,2}$ and Julia Vladimirova ${ }^{1 a}$ \\ ${ }^{1}$ M. V. Lomonosov Moscow State University, Lenin Hills, Moscow 119991, Russia \\ ${ }^{2}$ Institute of Spectroscopy, Russian Academy of Sciences, Fizicheskaya Str, 5, Troitsk, Moscow, 142190 Russia
}

Keywords: nanoparticle, near field, plasmonics, quantum emitter

Nonclassical behavior of light reveals already at the single-atom-level in resonance fluorescence both as the sub-Poissonian behavior of the photon-number statistics and as the phenomenon of antibunching of scattered photons [1]. The antibunching of photons is associated with two-photon correlation measurements and results from the fact that after emitting a photon an atom has to be reexcited in order to emit next photon. Therefore, the probability of joint detection of two photons increases with increasing the time interval between detections from zero to a lifetime of the atomic transition. The antibunching of photons in resonance fluorescence is opposite to that one in thermal beams, which show photon bunching. Antibunching is also closely related to sub-Poissonian behavior of the photon-number statistics, i.e., the distribution function of the emission probability of $n$ photons in a given time interval $T$. For the time intervals much longer than the atomic spontaneous decay time the sub-Poissonian behavior converges to a Gaussian one. First experimental confirmation of the antibunching of photons in resonance fluorescence was made in 1977 by Kimble, Dagenais, and Mandel [2] and the sub-Poissonian photon number statistics was later on verified by Short and Mandel in 1983 [3]. Most recent observation of the nonclassical properties of resonance fluorescent from a single trapped atomic ion was made by Diedrich and Walther [4].

Over two last decades, such a single-atom level of experiments for studying nonclassical light from isolated atoms or ions was also achieved in emerging field of nanophotonics [5], which demonstrates reliable sources of nonclassical light from single molecules, quantum dots (QD), NVcenters in nanodiamond, including those embedded in different nanostructures. In this case, the radiative properties of an emitter (atom, molecule or quantum dot) are strongly modified in confined geometries [6]. Plasmonic nanostructures, specifically metal nanoparticles (MNP), not only convert the incoming radiation to localized, but they also change the radiative frequency of the emitter and its decay rate. This leads to one of the important applications of nanooptics - using plasmonic nanostructures for changing and controlling fluorescence.

The resonance fluorescence, which features nonclassical behavior of fluorescent light, occurs when the quantum emitter is driven by an electromagnetic wave with the frequency close to the

\footnotetext{
${ }^{\mathrm{a}}$ Corresponding author: yu.vladimirova@physics.msu.ru
} 
emitter's resonant frequency [1] and studying the resonance fluorescence of a quantum emitter near a MNP is just at start [7] and no attention is paid to the nonclassical behavior of the scattered light.

In this talk, we study quantum-optical effects in the system of "quantum emitter (atom, molecule, quantum dot) + metal nanoparticle" driven by an external near-resonant laser field.

First, we will give an overview of the mechanisms of modification of the local field and radiative and nonradiative decay rates of the two-level atom located in close proximity to a metal nanoparticle. Simple analytical expressions are given for both the local field enhancement, the modified total decay rate of the atom near the metal nanosphere and the frequency shift of the atomic transition.

Then, we will analyze in more detail the polarization distribution at the nanoscale around the nanoparticle [9, 10]. Near-field being formed by the interference of the incident electromagnetic field with the local field of the nanoparticle strongly depends both on the frequency and polarization of the incident field. Dependence of the near-field intensity distribution on these parameters has been studied in detail, however how its polarization distribution depends on them still remains largely a challenging question, being crucial for experiments on interaction of a quantum emitter (atom, molecule, quantum dot) with plasmonic nanoparticles, which lies in the basis of a number of nowadays nanotechnologies. Specifically, we will study in detail the near-field polarization distribution of a plasmonic prolate nanospheroid in an incident electromagnetic field versus its polarization and frequency. Polarization properties of the near-field are described with the help of the 3D generalized Stokes parameters, which allow simple visualization. It is shown that this distribution has a complex structure, which drastically depends on the polarization of the incident laser field and on the parameters of the plasmon resonance of the nanoparticle. For the incident plane electromagnetic wave of arbitrary polarization, the nearfield of a prolate nanospheroid has areas with its polarization opposite to the polarization of the incident field. For instance, for the linearly polarized plane electromagnetic wave with the frequency tuned into the plasmon resonance of the nanoparticle the near-field polarization distribution features two symmetric tori in the center of which the near-field polarization is circular and vice versa: when the incident field polarization is circular, the near-field polarization distribution has a complex structure in the center of which the near-field polarization is linear. These results cover the whole set of particles whose shape varies from the spherical one to the nanoneedles and nanorods simply by changing the aspect ratio of the spheroid. A simple experiment is proposed to measure the near-field polarization distribution around a plasmonic nanoparticle [9].

After that, we will describe the photon-number statistics in resonance fluorescence of a two-level atom (quantum dot, molecule) near a MNP, which we consider for simplicity a metal nanosphere, driven by a laser field with finite bandwidth, as a function of atom's location around the nanoparticle, the intensity of the incident laser field, its bandwidth, detuning from the atomic resonance, and polarization. We have restricted ourselves to the two-level atom because we want to study the principal features of the nonclassical light from resonance fluorescence near a MNP. However, our approach can be also generalized to multilevel atoms in a manner analogous to the method applied for multilevel atoms in free space [8].

We will be shown that the distribution function $p(n, T)$ of the emission probability of $n$ photons in a given time interval $T$ strongly depends on the local field intensity in the point of space the atom is located at and can be controlled by a few key parameters - the atom's location around the metal nanosphere, the intensity of the incident laser field, its bandwidth, detuning from the atomic resonance, and polarization [11]. Analytical expressions for the mean and the variance of the number of photons are derived for the case when $T$ is much longer than the atomic transition lifetime. It is also shown that by analogy with the atom in free space [1], this distribution function showing subPoissonian character and antibunching of the emitted photons at short $T$ converges to a Gaussian one when $T$ is much longer than the modified by the nanoparticle natural lifetime of the excited atom. As a result, the typical convergence timescale becomes three orders of magnitude longer than for a free atom [11].

Finally, we will analyze the antibunching effect of photons from the resonance fluorescence in the system "metal nanosphere and a two-level atom" driving by the incident laser field [12].

In conclusion, we summarize the received results and discuss some possible applications. 
The authors acknowledge financial support from the Russian Foundation for Basic Research (grant No. 13-02-00446).

\section{References}

1. L. Mandel, E. Wolf, Optical Coherence and Quantum Optics (Cambridge Univ. Press, 1995)

2. H. J. Kimble, M. Dagenais, L. Mandel, Phys. Rev. Lett. 39, 691 (1977)

3. R. Short and L. Mandel, Phys. Rev. Lett. 51, 384 (1983)

4. F. Diedrich and H. Walther, Phys. Rev. Lett. 58, 203 (1987)

5. S.V. Gaponenko, Introduction to Nanophotonics (Cambridge Univ. Press, New York, 2010)

6. V.V. Klimov, Nanoplasmonics: Fundamentals and applications (Pan Stanford Publishing, Singapore, 2014)

7. Y.V. Vladimirova, V.V. Klimov, V.M. Pastukhov, and V.N. Zadkov, Phys. Rev. A 85, 053408 (2012)

8. Yu.V. Vladimirova, B.A. Grishanin, V.N. Zadkov, N.N. Kolachevskii, A.V. Akimov, N.A. Kisilev, S.I. Kanorskii, J. Exp. Theor. Phys. 96, 629 (2003)

9. E.D.Chubchev, Yu.V.Vladimirova, V.N.Zadkov, Optics Express 22, 20432 (2014)

10. E.D. Chubchev, Yu.V. Vladimirova, V.N. Zadkov, Laser Phys. Lett. 12, 015302 (2015)

11. V.M.Pastukhov, Yu.V.Vladimirova, V.N.Zadkov, Phys. Rev. A 90, 063831 (2014

12. V.M.Pastukhov, Yu.V.Vladimirova, V.N.Zadkov, submitted for publication (2015) 